\title{
An Empirical Force Field for the Simulation of the Vibrational Spectroscopy of Carbon Nanomaterials
}

\author{
Pritesh M. Tailor, Richard J. Wheatley and Nicholas A. Besley* \\ School of Chemistry, University of Nottingham, University Park, Nottingham, NG7 2RD, \\ $U K$
}

\begin{abstract}
An empirical force field for carbon based upon the Murrell-Mottram potential is developed for the calculation of the vibrational frequencies of carbon nanomaterials. The potential is reparameterised using data from density functional theory calculations through a Monte-Carlo hessian-matching approach, and when used in conjunction with the empirical bond polarisability model provides an accurate description of the non-resonant Raman spectroscopy of carbon nanotubes and graphene. With the availability of analytical first and second derivatives, the computational cost of evaluating harmonic vibrational frequencies is a fraction of the cost of corresponding quantum chemical calculations, and makes the accurate atomistic vibrational analysis of systems with thousands of atoms possible. Subsequently, the non-resonant Raman spectroscopy of carbon nanotubes and graphene, including the role of defects and carbon nanotube junctions is explored.
\end{abstract}

Keywords: Raman spectroscopy, force field, carbon nanotubes, graphene

\section{Introduction}

Since their discovery, carbon nanotubes (CNTs) [1] and graphene [2] have been the subject of considerable research interest owing to their unique structural, mechanical and electronic properties. Raman spectroscopy has emerged

\footnotetext{
${ }^{1}$ nick.besley@nottingham.ac.uk (Nicholas Besley)

${ }^{2}+441159513474$ (Nicholas Besley)
}

Preprint submitted to Carbon

November 18, 2016 
5 as one of the most popular techniques used to study these systems, providing information on the structure, bonding and environment of CNTs [3] and graphene [4], including details on CNT diameter, chirality and defects. Key modes in the Raman spectroscopy of CNTs are the G band and the radial breathing mode $(\mathrm{RBM})$. The $\mathrm{G}$ band arises from planar vibrations of the carbon atoms and is 10 the most intense band, and is present in both graphene and CNTs. For CNTs this band can split into the $\mathrm{G}^{+}$and $\mathrm{G}^{-}$bands, which correspond to in-plane movements along and perpendicular to the CNT axis, respectively. The G band can be used to determine whether a CNT is metallic or semi-conducting, and allows for the qualitative assignment of the chiral indices of a CNT. The RBM corresponds to the coherent expansion and contraction of carbon atoms in the radial direction, and the frequency of this mode is known to depend on the diameter of the CNT. A further very weak band is the disorder-induced D band, which is associated with $s p^{3}$ defects in CNTs [5].

Although Raman spectroscopy is used routinely to study CNTs and graphene based materials, the prediction of the Raman spectroscopy of these systems is a challenge for computational methods. The accurate calculation of the vibrational frequencies and associated spectroscopy of carbon nanomaterials can potentially aid the interpretation of experimental measurements and allow the relationship between the molecular structure and the observed features to be explored. Simulation of vibrational spectroscopy using quantum chemical methods, such as density functional theory (DFT), is well established. However, the computational cost of these methods makes them impractical for the study of large systems such as CNTs. This can be overcome for the simulation of pristine nanotubes through the use of periodic boundary conditions, but the use of periodic boundary conditions to study realistic CNTs that are non-uniform and contain vacancies and defects is limited since such calculations would require large unit cells. Furthermore, periodic boundary conditions are not suitable for studying systems such as nanotube junctions which are inherently non-periodic. 
Several groups have reported DFT based harmonic frequency calculations of the infrared (IR) and Raman spectroscopy of fullerenes, including $\mathrm{C}_{60}$ and $\mathrm{C}_{70}$ 6, 7, 8, 9, 10, Similar calculations have also been performed for related systems such as [n]cycloparaphenylenes [11, 12] and a nanotorus [13]. DFT calculations 40 have been used to study the Raman spectroscopy of zigzag nanotubes of varying diameter [14], accurately reproducing the diameter dependence of the RBM, although these calculations only considered one unit cell capped with hydrogen atoms. Such small model systems would not be expected to describe other bands such as the G band accurately. Another study employed DFT to study the vibrational spectroscopy of ultra-small diameter nanotubes [15. Yumura et al. reported DFT calculations of the vibrational frequencies of relatively large nanotubes, consisting of up to 120 carbon atoms, and studied the effects of the end caps on the spectra [16. Recently the Raman spectroscopy of zigzag nanotubes with length of up to $2.15 \mathrm{~nm}$ was studied using DFT, with larger tubes studied using a cartesian coordinate transfer technique [17. The calculations reproduced the dependence of the frequency of the RBM on the tube diameter, but larger tubes were required to describe the $\mathrm{G}$ band modes accurately. It was found that the $\mathrm{G}$ band was more sensitive to the presence of defects, and there was a reduction in the intensity of the RBM on the introduction of a Stone-Wales (SW) defect. These studies represent the current limit of what can be achieved through direct non-periodic DFT calculations. The Raman spectroscopy of nanotubes including the effect of SW and di-vacancy defects on the spectra has been studied [18] using plane-wave based periodic DFT with the Raman intensities computed using the empirical bond polarisation model (BPM) 19]. The BPM is an example of a computationally inexpensive approach to evaluating Raman intensities and other related approaches have been reported and used to study the Raman spectra of liquids [20]. Other work has studied the effect of the Raman excitation profile for CNTs under torsion or uniaxial strain 21. Computationally less expensive methods that have been applied to study the vibrational spectroscopy of CNTs include tight-binding DFT, which has been applied to study the vibrational spectra of fullerenes [22, 23, and force 
constant based methods [24, 25, 26, 27]. Calculations of the resonant Raman spectroscopy of CNTs using a non-orthogonal tight-binding model have been reported, and the influence of point defects on the spectra studied [28, 29].

Ideally it would be possible to model the vibrational spectroscopy of these systems efficiently without the constraints of periodicity, providing an accurate description of experiment and opening the opportunity for the role of edges and defects to be explored. Empirical potentials provide a possible solution to this problem. The attraction of this approach for studying the vibrational spectroscopy of these systems is evident by comparing the associated computational cost with a DFT calculation. On a single processor, a B3LYP/6-31G* calculation of the harmonic frequencies of $\mathrm{C}_{60}$ and $\mathrm{C}_{70}$ requires over 40000 and 60000 seconds, respectively. Similar calculations using the empirical Murrell-Mottram potential take two and three seconds, representing a many thousand-fold decrease in computational time. Furthermore, the use of empirical potentials alleviates the memory requirements of using quantum chemical methods, allowing systems of thousands of atoms to be studied routinely. However, for empirical potentials to form viable methods for simulating the vibrational spectroscopy of these systems, the accuracy of quantum chemical methods needs to be retained.

The prominence of carbon-carbon interactions in chemistry, physics and materials science has led to the introduction of several highly developed empirical potentials. Bond order potentials that can describe changes in hybridization and the breaking and formation of bonds are one of the most popular types of potential. These include the Reactive Empirical Bond Order (REBO) potential [30, which is a development of the Brenner potential [31] which was based on the Tersoff potential [32, and more recently the Reactive Force Field (Reaxff) potential was introduced [33]. The Raman spectroscopy of nanotube 95 junctions have been studied based upon the REBO potential [34. Several other simpler potentials for carbon have been reported in the literature including the TLHT potential of Takai et al. [35] and the potentials of Murrell and co-workers 
[36, 37, 38. Within the context of computing vibrational spectroscopy, one of the advantages of these mathematically simpler potentials is that it is relatively straightforward to implement analytical first and second derivatives. The availability of analytical second derivatives is essential for the efficient calculation of vibrational spectroscopy and, owing to the mathematical complexity, generating analytical second derivatives for the bond order based potentials represents a significant challenge. Furthermore, much of the complexity of the reactive potentials is not relevant for the calculation of vibrational frequencies, which are inherently an equilibrium property.

In this work we study the vibrational spectroscopy of carbon based materials using empirical potentials. The parameterisation of the well known potentials, such as the REBO potential [30], focuses on energetics and structure and does not consider the vibrational properties. As a consequence of this, it has been shown in a recent study that the REBO potential performed poorly in describing the the vibrational frequencies of $\mathrm{C}_{60}$ and CNTs [39. It was found that to achieve an adequate description of the IR spectrum of $\mathrm{C}_{70}$ and the RBM and $\mathrm{G}$ band of CNTs, it was necessary to treat high and low frequency modes separately. The calculation of vibrational frequencies has implications beyond simulating IR and Raman spectra. Vibrational frequencies provide an indirect probe of the potential energy surface, providing a measure of the curvature of the surface, and it is an open question as to whether empirical potentials which do not produce satisfactory agreement with experimental frequencies can provide accurate molecular dynamics.

Here we develop an empirical potential based upon the Murrell-Mottram (MM) potential that is designed to predict the vibrational frequencies of carbon 125 based materials. Analytical first and second derivatives of the MM potential have been implemented and the potential reparameterised via a Monte-Carlo based hessian-matching approach. When combined with the empirical bond polarisation model [19] it provides a fully empirical method for modelling the 
Raman spectroscopy of CNTs and graphene. The implementation of analytical first and second derivatives allows accurate atomistic vibrational analysis and simulation of the Raman spectroscopy of systems with greater than 10,000 atoms. Subsequently, the Raman spectroscopy of graphene and CNTs with defects and junctions is explored.

\section{Computational Details}

\subsection{Parameterisation of the Murrell-Mottram Potential}

The work presented in this study is based upon the MM potential. This potential was chosen in preference to bond order potentials like REBO and Reaxff for its simplicity that allows for the implementation of analytical first and second derivatives and a more straightforward framework for parameterisation.

For a system with $N$ atoms, the MM potential [36] takes the following form with two and three body terms:

$$
E=\sum_{i<j}^{N} V_{i j}^{(2)}+\sum_{i<j<k}^{N} V_{i j k}^{(3)}
$$

where

$$
\begin{aligned}
V_{i j}^{(2)} & =-D\left(1+a_{2} \rho_{i j}\right) \exp \left(-a_{2} \rho_{i j}\right) \\
V_{i j k}^{(3)} & =-D P\left(Q_{1}, Q_{2}, Q_{3}\right) \exp \left(-a_{3} Q_{1}\right) \\
\rho_{i j} & =\left(r_{i j}-r_{e}\right) / r_{e}
\end{aligned}
$$

with $r_{i j}$ being the distance between atoms $i$ and $j . D$ and $r_{e}$ are parameters that allow the energy and ground state structure to be described accurately. The $\exp \left(-a_{2} \rho_{i j}\right)$ and $\exp \left(-a_{3} Q_{1}\right)$ terms are damping functions that ensure the energy goes to zero as the atoms become infinitely separated with the form of the function controlled by the parameters $a_{2}$ and $a_{3} . P\left(Q_{1}, Q_{2}, Q_{3}\right)$ is a quartic polynomial in symmetrical coordinates $Q_{i}$ which has the following form

$$
\begin{aligned}
P\left(Q_{1}, Q_{2}, Q_{3}\right) & =c_{0}+c_{1} Q_{1}+c_{2} Q_{1}^{2}+c_{3}\left(Q_{2}^{2}+Q_{3}^{2}\right)+c_{4} Q_{1}^{3}+c_{5} Q_{1}\left(Q_{2}^{2}+Q_{3}^{2}\right) \\
& +c_{6}\left(Q_{3}^{3}-3 Q_{3} Q_{2}^{2}\right)+c_{7} Q_{1}^{4}+c_{8} Q_{1}^{2}\left(Q_{2}^{2}+Q_{3}^{2}\right) \\
& +c_{9}\left(Q_{2}^{2}+Q_{3}^{2}\right)^{2}+c_{10} Q_{1}\left(Q_{3}^{3}-3 Q_{3} Q_{2}^{2}\right)
\end{aligned}
$$


where $c_{0}-c_{10}$ are parameters and

$$
\begin{aligned}
Q_{1} & =\frac{1}{\sqrt{3}}\left(\rho_{i j}+\rho_{i k}+\rho_{j k}\right) \\
Q_{2} & =\frac{1}{\sqrt{2}}\left(\rho_{i k}-\rho_{j k}\right) \\
Q_{3} & =\frac{1}{\sqrt{6}}\left(2 \rho_{i j}-\rho_{i k}-\rho_{j k}\right) .
\end{aligned}
$$

150 computed from quantum chemical calculations by a Monte-Carlo minimisation of the root mean square deviation (RMSD) between the forces [48, 49]. Recently, the use of hessian data in the parametisation of force fields has been exploited 
175 [50.

New parameters for the MM potential were determined by a Monte-Carlo based minimization of the RMSD of the unscaled hessian matrix for $\mathrm{C}_{60}$, computed using DFT with the B3LYP exchange-correlation functional [51, 52] and 6- $31 \mathrm{G}^{*}$ basis set within the constraint that a low RMSD for the structure is also achieved. $\mathrm{C}_{60}$ was chosen since it captures many of the structural elements present in $s p^{2}$-based carbon materials, and it is sufficiently small for the calculation of the hessian matrix using DFT. Previous studies have shown that the B3LYP $/ 6-31 \mathrm{G}^{*}$ level of theory provides a good description the structure and vibrational frequencies of fullerenes 7 . In the fitting, the parameter $D$ was not varied from the value of $6.298 \mathrm{eV}$ from the work of Eggen et al. [38] where this value corresponds to the typical bond energy of a carbon-carbon double bond. The $c_{i}$ parameters were confined to the range -200 to +200 , the $a_{i}$ parameters between 1.5 and 10.0. $r_{e}$ was allowed to vary between 1.1 and $1.5 \AA$ since this range contains acceptable values for the bond lengths of $\mathrm{C}_{60} .10000$ sets of randomly chosen initial parameters were generated, and then for each of these sets of parameters, the RMSD ${ }^{\text {structure }}$ and $\mathrm{RMSD}^{\text {hessian }}$ were minimised using the following procedure: Each parameter is perturbed randomly by an amount between -5 and +5 for the $c_{i}$ coefficients and between -0.05 and 0.05 for the remaining parameters, and the change is accepted if both the RMSD ${ }^{\text {structure }}$ and RMSD ${ }^{\text {hessian }}$ decrease. This was continued until there was no further decrease in RMSD ${ }^{\text {structure }}$ and RMSD hessian, and was repeated three times for each starting condition. The 1200 sets of parameters with lowest RMSD ${ }^{\text {hessian }}$ that satisfied the condition RMSD ${ }^{\text {structure }}<0.03 \AA$ were refined further using a similar procedure with the magnitude of the variation in the parameters reduced by a factor of 100 . The final results showed several sets of parameters with similarly low RMSD ${ }^{\text {structure }}$ and $\mathrm{RMSD}^{\text {hessian }}$, these parameters were then tested in the simulation of a Raman spectrum for a nanotube. The final parameters chosen are shown in Table 1 and correspond to RMSD ${ }^{\text {structure }}=$ ${ }_{205} 0.014 \AA$ and $\mathrm{RMSD}^{\text {hessian }}=0.37 E_{h} \AA^{-2}$. There is a significant reduction 
in the value of $r_{e}$ between the original MM potential for carbon and the new parameters reflecting the change from $s p^{3}$ to $s p^{2}$ carbons and the presence of carbon-carbon double bonds. Some of the $c$ coefficients change significantly, however, the potential depends on the cumulative effect of these parameters and it is difficult to determine any physical relevance from the change in any one of these coefficients. The RMSD in the hessian is significant, but as shown later this is sufficiently low to provide an accurate description of the vibrational frequencies of CNTs and graphene. The vibrational frequencies are computed within the harmonic approximation, and it is well known that this leads to an overestimation of the vibrational frequencies. Consequently, it is customary to apply a linear scaling to computed harmonic frequencies. For B3LYP/6-31G* a scaling factor of about 0.96 is optimal [53, for the frequencies computed with the re-parameterised MM potential we find a scaling factor of 0.90 to be suitable which is closer to the scaling factors used for Hartree-Fock theory calculations. We note that overestimating the experimental frequencies at the harmonic level is to be expected since if the calculations were extended to anharmonic frequencies, for example via vibrational perturbation theory, this would lead to a decrease in the computed frequencies.

\subsection{Raman Intensities}

To simulate Raman spectra it is necessary to combine the computed frequencies with Raman intensities associated with the corresponding normal modes. Within a non quantum chemical framework, this can be achieved using the BPM. The BPM has been previously used for the computation of Raman spectra of CNTs, graphene and nano-ribbons [54, 55], and provides a complementary empirical approach for estimating Raman intensities. One limitation of the model is that it cannot be applied to resonant Raman spectra. The BPM has been used in conjunction with periodic calculations using a force-constant model [54, 55]. and DFT [18, thus avoiding the computationally expensive determination of the polarisability derivatives via quantum chemical calculations. 


\begin{tabular}{lll}
\hline Parameter & $\mathrm{MM}^{\text {Eggen }}$ & $\mathrm{MM}^{\text {Vib }}$ \\
\hline$D / \mathrm{eV}$ & 6.298 & 6.298 \\
$r_{e} / \AA$ & 1.570 & 1.313 \\
$a_{2}$ & 8.200 & 7.428 \\
$a_{3}$ & 8.200 & 8.072 \\
$c_{0}$ & 8.087 & 7.788 \\
$c_{1}$ & -13.334 & 3.917 \\
$c_{2}$ & 26.882 & -17.503 \\
$c_{3}$ & -51.646 & -51.427 \\
$c_{4}$ & 12.164 & 99.263 \\
$c_{5}$ & 51.629 & -39.772 \\
$c_{6}$ & 25.697 & 70.505 \\
$c_{7}$ & -5.964 & 73.262 \\
$c_{8}$ & -7.306 & 3.831 \\
$c_{9}$ & 2.208 & 65.696 \\
$c_{10}$ & 13.707 & -85.307 \\
\hline
\end{tabular}

Table 1: Parameters for the Murrell-Mottram potential from the work of Eggen et al. $\left(\mathrm{MM}^{\text {Eggen }}\right)$ and the new parameters determined in this work $\left(\mathrm{MM}^{V i b}\right)$. 
Within the BPM, the bond polarisability for a pair of atoms is given as

$$
\Pi_{\mu \nu}=\frac{1}{3}\left(\alpha_{\|}+2 \alpha_{\perp}\right) \delta_{\mu \nu}+\left(\alpha_{\|}-\alpha_{\perp}\right)\left(\frac{R_{\mu} R_{\nu}}{R^{2}}-\frac{1}{3} \delta_{\mu \nu}\right)
$$

where $\mu$ and $\nu$ are Cartesian coordinates and $\mathbf{R}$ is the vector connecting the two atoms linked by the bond. Raman intensities can be calculated from derivatives of equation 9 with respect to the normal modes, and equations for these derivatives can be found elsewhere [19, 25. $\left(\alpha_{\|}+2 \alpha_{\perp}\right)$ and $\left(\alpha_{\|}-\alpha_{\perp}\right)$ and the associated derivatives $\left(\alpha_{\|}^{\prime}+2 \alpha_{\perp}^{\prime}\right),\left(\alpha_{\|}^{\prime}-\alpha_{\perp}^{\prime}\right)$ are empirical parameters. Here we adopt the parameters used by Saito et al., for modelling CNTs [55] and graphene [54]. For CNT $\left(\alpha_{\|}^{\prime}+2 \alpha_{\perp}^{\prime}\right)=4.7 \AA^{2},\left(\alpha_{\|}^{\prime}-\alpha_{\perp}^{\prime}\right)=4.0 \AA^{2}$ and $\left(\alpha_{\|}-\alpha_{\perp}\right)=0.04 \AA^{3}$ and for graphene $\left(\alpha_{\|}^{\prime}+2 \alpha_{\perp}^{\prime}\right)=7.55 \AA^{2},\left(\alpha_{\|}^{\prime}-\alpha_{\perp}^{\prime}\right)=$ $2.60 \AA^{2}$ and $\left(\alpha_{\|}-\alpha_{\perp}\right)=0.32 \AA^{3}$. We note that these parameters were designed for the study of CNTs under periodic boundary calculations, and as a result may not be ideal for the calculations presented here. Furthermore, it would be desirable to have a common set of parameters for CNTs and graphene.

\subsection{Calculations}

Using the new MM potential, structures were optimized using the conjugate gradient method with a gradient convergence criterion of $10^{-8} E_{h} \AA^{-1}$, and a spherical cut off was applied to the potential with a radius of $12 \AA$ with an update every 200 steps during optimization. Harmonic vibrational frequencies and normal modes were calculated through diagonalisation of the mass-weighted hessian matrix. The elements of the hessian matrix were constructed using analytical derivatives with the same spherical cut off of $12 \AA$. Analytical derivatives are critical for efficient structure and frequency calculations, and for $\mathrm{C}_{180}$ the computational cost is over 3000 times less expensive than the use of numerical derivatives based upon energies, and this difference grows rapidly as the system size increases. Furthermore, ambiguity regarding the step size within the numerical derivative methods is removed. Subsequently, the vibrational modes were visualized in either IQMOL or VMD. Isotopic effects of including ${ }^{13} \mathrm{C}$ in naturally occurring abundances were explored and showed to have negligible 


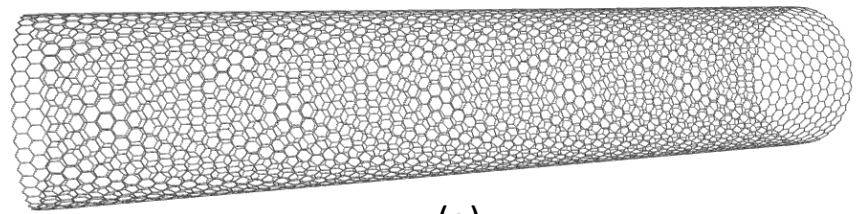

(a)

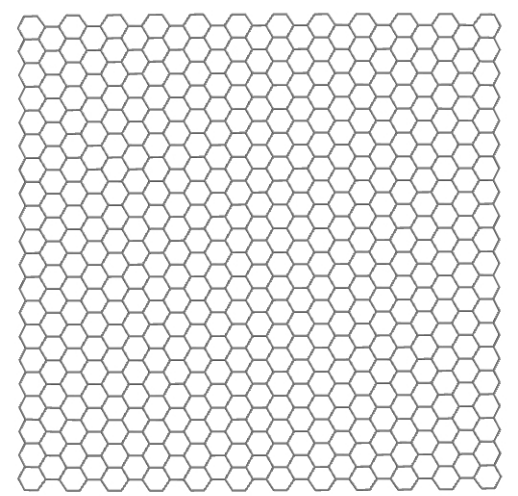

(b)

Figure 1: Optimized structures of a $(36,0)$ nanotube of length $15.2 \mathrm{~nm}$ (a) and $5 \mathrm{~nm}$ x $5 \mathrm{~nm}$ graphene flake (b).

effects on the computed spectra. Non-resonant Raman intensities via the BPM were convoluted with Lorentzian functions with FWHM's of $20 \mathrm{~cm}^{-1}$, unless stated otherwise. DFT calculations were performed using the Q-CHEM software [56] and the calculations using the MM potential and BPM were performed with our own code.

\section{Results and Discussion}

\subsection{Structure}

The new parameters of the potential find CNTs and graphene to be stable structures as demonstrated by the absence of imaginary frequencies, and representative structures are shown in Figure 1. The previous parameterisation of the MM potential for carbon did not produce $s p^{2}$ carbon as stable structures,

${ }_{275}$ but rather tetrahedrally distorted coordination of carbon atoms in a tubular 
arrangement. This is unsurprising since the parameters were fitted to the properties of diamond. For nanotubes with diameters greater than that of a $(10,10)$ nanotube, the predicted C-C bond lengths are $1.43 \AA$, consistent with a value of approximately $1.427 \AA$ observed in experiment. For small diameter nanotubes the strain arising for the high curvature results in greater deviation in the bond lengths. For graphene the new potential gives bond lengths of $1.42 \AA$ at the center of a model $5 \mathrm{~nm} \times 5 \mathrm{~nm}$ flake, again this compares well to literature values. At the edges of the flake, bonds lengths deviate from $1.42 \AA$ with edge effects becoming more prominent and differentiation between the bond lengths of double and single bonds is evident. Overall, this shows that the parameters fitted to $\mathrm{C}_{60}$ are able to provide a reliable description of the minimum energy structures of large CNTs and graphene.

\subsection{Raman Spectra}

Figure 2 shows the Raman spectrum computed with the empirical model for a single CNT along with an experimental spectrum reported by Dresselhaus and co-workers [57]. The calculation is for a $4 \mathrm{~nm}$ long $(12,0)$ nanotube. The theoretical spectrum predicts the RBM, intermediate frequency band (IFM) and $\mathrm{G}$ bands that are observed in experiment. The D band is associated with defects $/ \mathrm{sp}^{3}$ environments which are not present in the pristine CNT modelled here and overtone bands at higher frequency than the $\mathrm{G}$ band observed in experiment are not present in the fundamental harmonic frequency analysis presented here. The most intense band is the $\mathrm{G}$ band which lies at $1608 \mathrm{~cm}^{-1}$ in the theoretical spectrum compared with about $1590 \mathrm{~cm}^{-1}$ in experiment. The profile of the $\mathrm{G}$ band shows two distinct peaks, and this is discussed in more detail later. The predicted frequencies for the RBM and IFM are in good agreement with the experimental spectrum, although direct comparison with the experiment for these bands is problematic since the frequencies of these bands are sensitive to the diameter of the nanotube and the precise diameter of the tubes in experiment is not well defined. The main difference between the computed and experimental spectra is the underestimation of the intensities of the RBM 

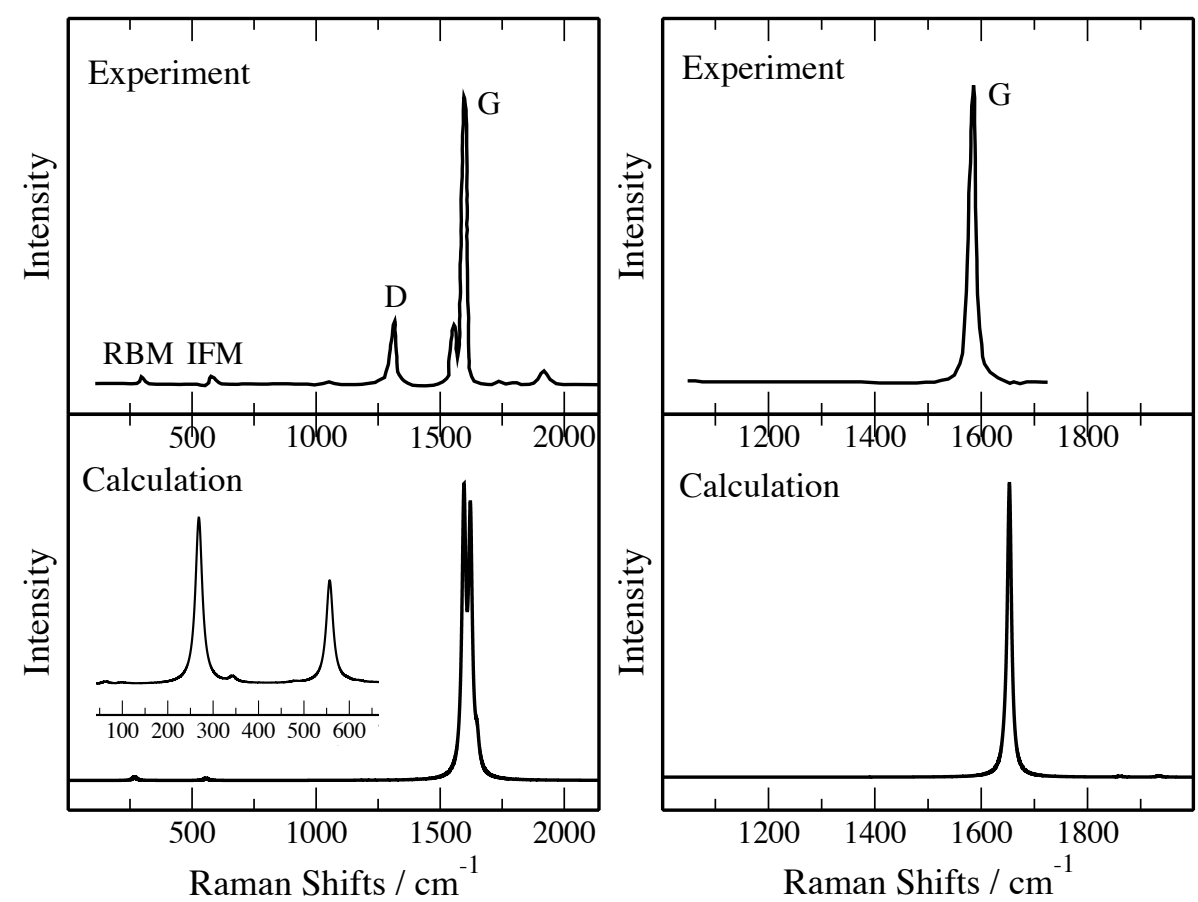

Figure 2: Experimental and computed Raman spectra for a carbon nanotube (left) and graphene (right). Calculations are for a $(12,0)$ nanotube of length $4 \mathrm{~nm}$, and the experimental spectrum is adapted from reference [57].

and IFM bands relative to the $\mathrm{G}$ band. One noticeable feature of the RBM for finite nanotubes is that there is some distortion to the perfect radial expansion at the ends of the tube, while within a periodic (infinite) tube framework, the displacements of the atoms are perfectly radial and symmetrical. A consequence of this is that the Raman intensity associated with the RBM for the finite tube will be smaller and is likely to be underestimated by the BPM parameters that were parameterised based upon periodic (infinite tube) calculations.

Also shown in Figure 2 are corresponding spectra for graphene. The calculations correspond to a finite $5 \mathrm{~nm} \times 5 \mathrm{~nm}$ graphene sheet. There are fewer features in Raman spectra for graphene, and the spectra are dominated by the G band. The calculations predict a spectrum in agreement with experiment, 


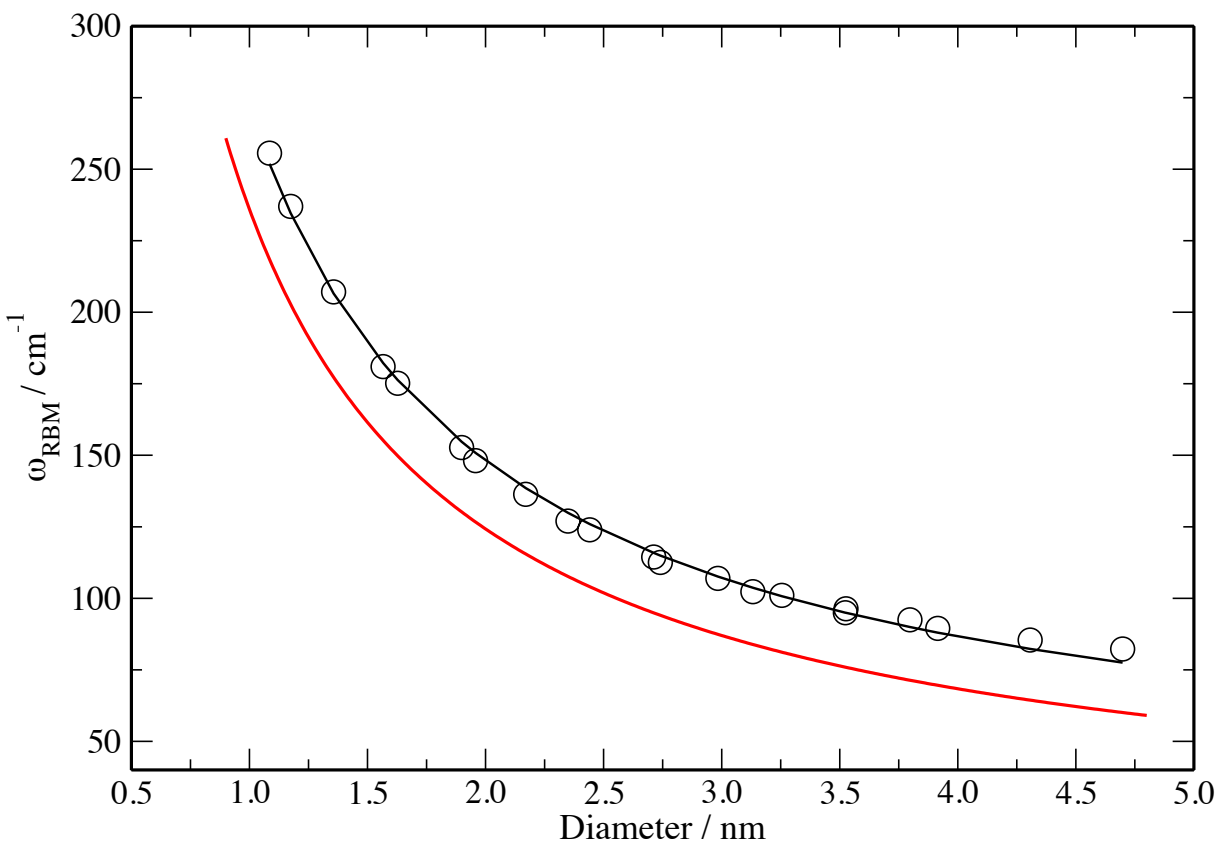

Figure 3: Variation of the $\omega_{R B M}$ with nanotube diameter. Circles: calculated frequencies, black line: $\omega_{R B M}=\frac{234}{d}+25$, red line: $\omega_{R B M}=\frac{223.5}{d}+12.5$.

the frequency of the $\mathrm{G}$ band is calculated to be about $1650 \mathrm{~cm}^{-1}$ compared with $1585 \mathrm{~cm}^{-1}$ in experiment. The agreement with experiment for this band is not as accurate as found for the nanotube and may reflect the nature of the fitting of the potential to $\mathrm{C}_{60}$, which is a more appropriate model for nanotubes than for a flat graphene sheet.

\subsection{Radial Breathing Mode}

The RBM is one of the most important modes in the Raman spectroscopy of CNTs. The primary use of this mode stems from the relationship of its frequency to the diameter of the nanotube and the RBM is commonly used to determine nanotube diameters in experimental samples. The frequency of the RBM is known to follow an inverse relationship with the diameter $(d)$ of the 
$\mathrm{CNT}$, and is commonly used to determine nanotube diameters in experimental samples. The following empirical relationships are often used [58, 59]

$$
\begin{aligned}
\omega_{R B M} & =\frac{A}{d} \\
\omega_{R B M} & =\frac{A}{d}+B
\end{aligned}
$$

For $\omega_{R B M}$ in $\mathrm{cm}^{-1}$ and $d$ in nm, $A$ is known to lie between $223-248 \mathrm{~nm} \mathrm{~cm}^{-1}$, and $B$ typically has values $0-20 \mathrm{~cm}^{-1}[58,59,60$. Figure 3 shows the variations of the RBM frequency computed with the new potential with diameter for a set of nanotubes comprising both armchair and zigzag CNTs. Fitting of these frequencies to equation 10 gives a value of $A=234 \mathrm{~nm} \mathrm{~cm}^{-1}$ and to equation 11 gives values of $A=234 \mathrm{~nm} \mathrm{~cm} \mathrm{~cm}^{-1}$ and $B=25 \mathrm{~cm}^{-1}$, and in both cases the correlation coefficient is 0.999 . Also shown are the RBM frequencies given by a commonly used empirical formula $\omega_{R B M}=\frac{223.5}{d}+12.5$. This shows that 340 the potential accurately describes the diameter dependence of the RBM mode observed in experiment, although the frequencies are a little too high. The calculations also show that the frequency of the RBM converges rapidly with the length of the tube and for nanotubes longer than $10 \mathrm{~nm}$ there is little change in the computed RBM frequency.

\section{4. $G$ Band}

In nanotubes the $\mathrm{G}$ band is known to have two components, $\mathrm{G}^{-}$and $\mathrm{G}^{+}$. $\mathrm{G}^{-}$lies at lower frequency and corresponds to a vibrational mode wherein the carbons are moving perpendicular to the nanotube axis, and this mode is more sensitive to the diameter of the nanotube. $\mathrm{G}^{+}$lies at higher frequency and corresponds to carbon motions along the tube axis. The splitting between these modes can lead to two bands and can distinguish between metallic and semiconducting tubes. Figure 4 shows the computed $\mathrm{G}$ band for two nanotubes of different diameter, $(8,8)$ and $(12,12)$ with lengths of $15 \mathrm{~nm}$. The nature of the modes comprising the $\mathrm{G}$ band are predicted correctly. For both nanotubes the 


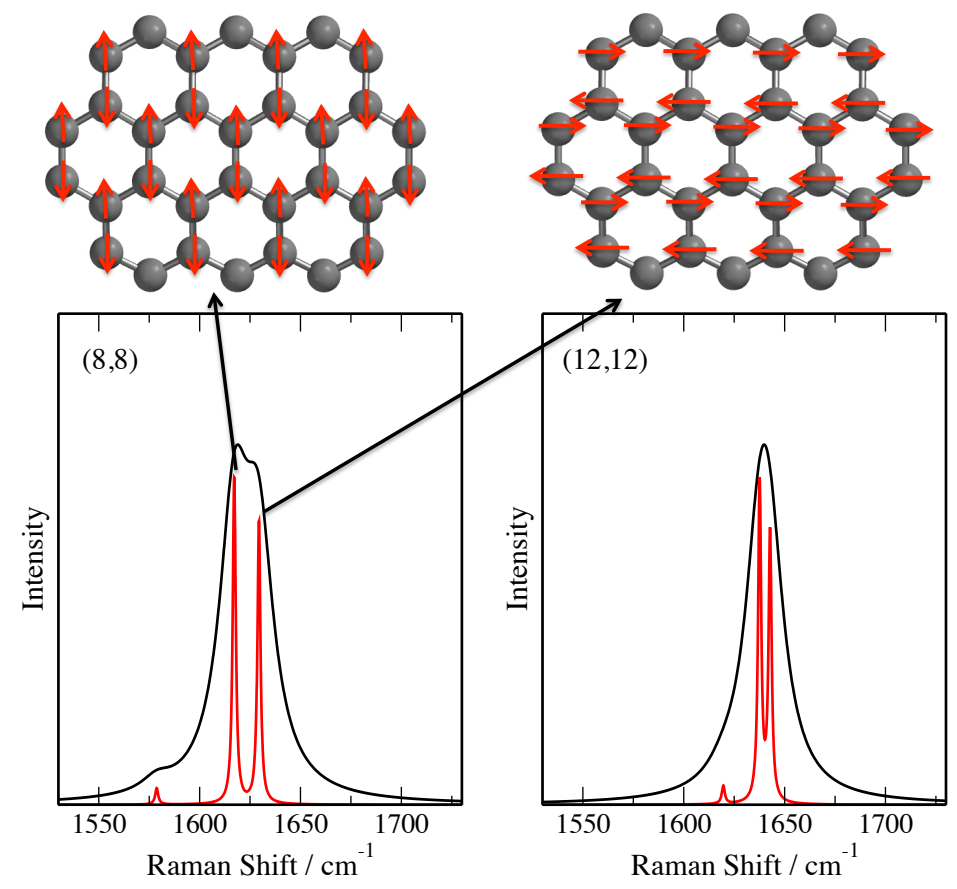

Figure 4: Computed $\mathrm{G}$ band for $(8,8)$ and $(12,12)$ nanotubes, and illustration of the $\mathrm{G}^{-}$and $\mathrm{G}^{+}$modes. Black line: Lorentzian broadening with width $20 \mathrm{~cm}^{-1}$, red line: Lorentzian broadening with width $2 \mathrm{~cm}^{-1}$

$\mathrm{G}$ band has two distinct components, and observation of the corresponding normal modes shows that the lower frequency mode has predominately motions of the carbons perpendicular to the tube axis and the higher frequency mode has motions along the nanotube axis. The frequency of both components changes with the diameter of the nanotube, and this is illustrated in Figure 5 which shows the variation of the frequency of the $\mathrm{G}^{-}$and $\mathrm{G}^{+}$modes with nanotube diameter. The $\mathrm{G}^{-}$mode is more sensitive to diameter, and as the tube diameter increases the two components of the $\mathrm{G}$ band become closer in frequency. The computed band profiles with the Lorentzian broadening of $20 \mathrm{~cm}^{-1}$ do not show two clearly distinct peaks. Armchair nanotubes, such as the ones modelled here, are metallic and two peaks are expected for semiconducting nanotubes. The frequency of the $\mathrm{G}$ band does not show a strong dependence on the length 
of the CNT. This is not surprising since the G band is common across a wide range of carbon nanomaterials, hence its main properties are independent of large-scale structural differences such as that between CNT and graphene.

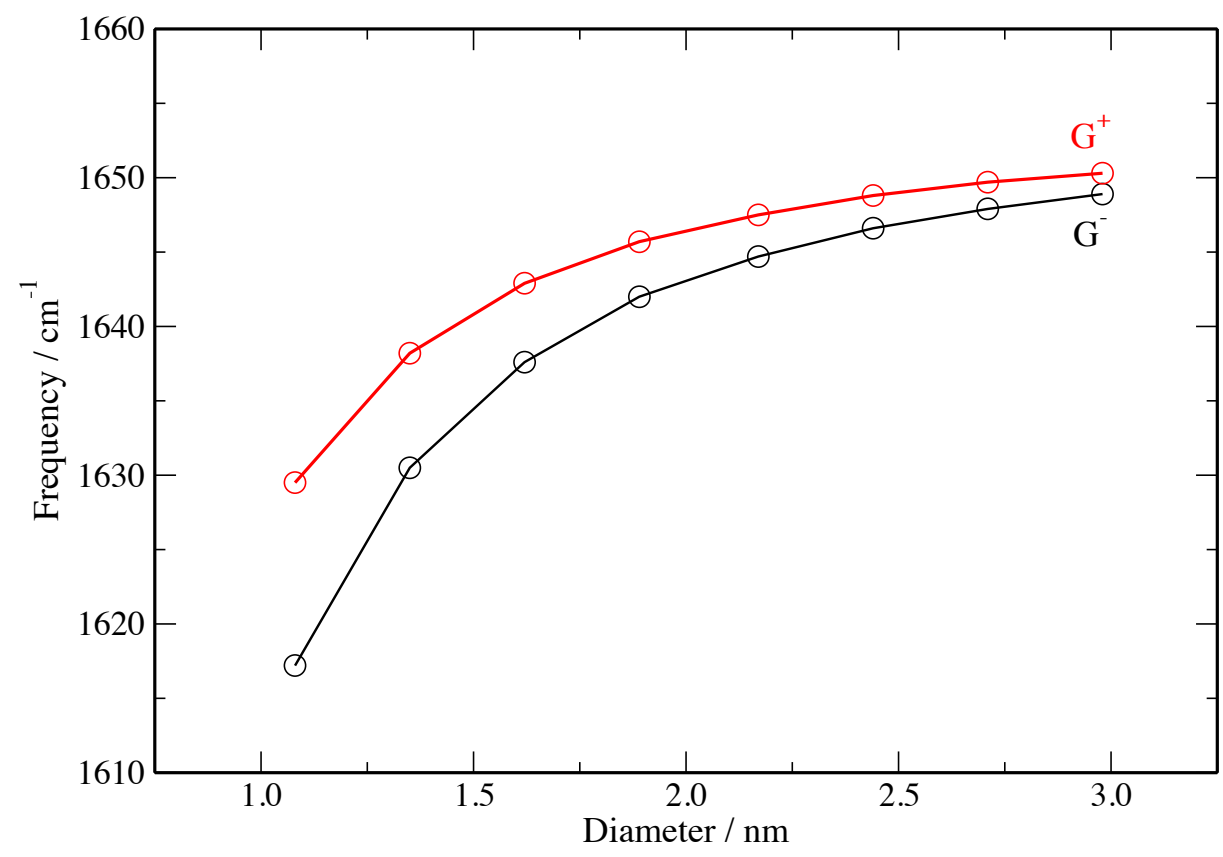

Figure 5: Variation of the frequency of the $\mathrm{G}^{+}$and $\mathrm{G}^{-}$bands with nanotube diameter.

\subsection{Vibrational Density of States}

Calculations of Raman spectra only probe the small number of vibrational modes that have significant Raman intensity. The vibrational density of states (VDOS) provides a measure of the complete vibrational spectrum. For fi375 nite nanotubes of sufficient length the calculated VDOS can approach bulk behaviour. Figure 6 shows the computed VDOS for a $(10,10)$ nanotube with length $60 \mathrm{~nm}$ that has 9720 atoms, and a broadening using Lorentzian functions of $20 \mathrm{~cm}^{-1}$ is applied. This can be compared with the VDOS measured in experiment from the work of Dresselhaus and Eklund [61 which also corresponds 380 to $(10,10)$ nanotubes. We note that the calculations represent a single pristine 


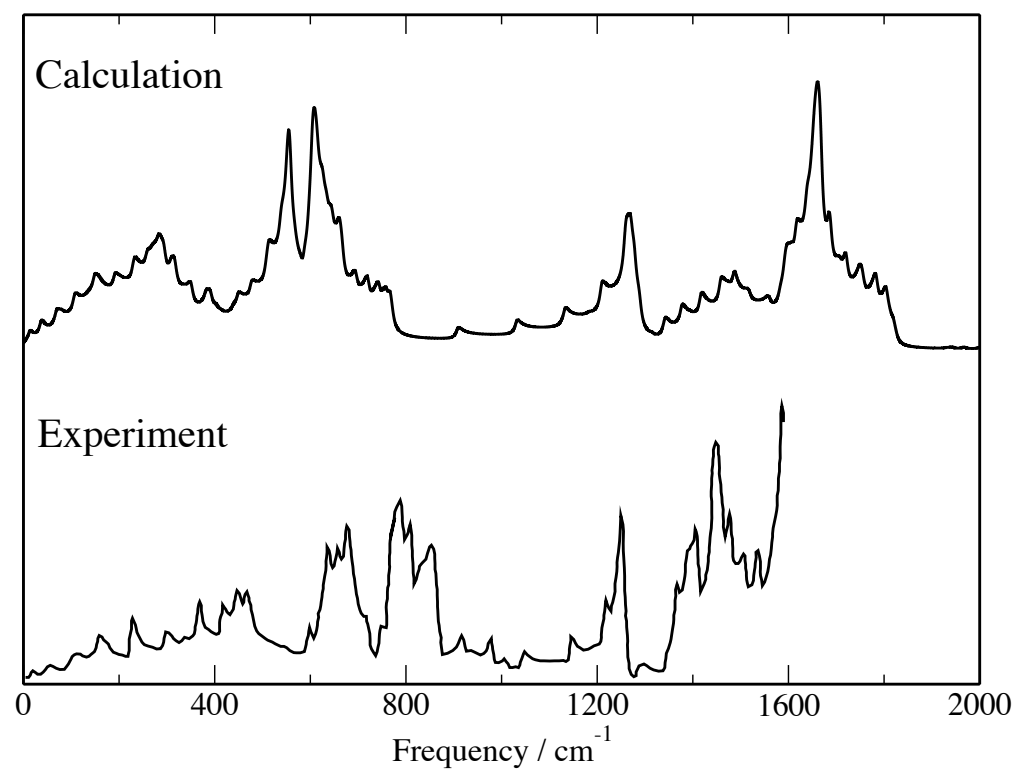

Figure 6: Calculated and experimental vibrational density of states. Experimental data from reference 61.

CNT while the experiments are for a nanotube bundle. In general the calculations qualitatively reproduce the VDOS profile observed in experiment. In some regions of the spectrum some notable differences are revealed. In particular the two distinct peaks in the $600 \mathrm{~cm}^{-1}$ to $900 \mathrm{~cm}^{-1}$ region are predicted to lie at lower frequency. There are also differences in the $1400 \mathrm{~cm}^{-1}$ to $1600 \mathrm{~cm}^{-1}$ region. However, the potential is performing reasonably well over the frequency range, although some features are slightly shifted.

\subsection{Stone-Wales Defects}

The Stone-Wales (SW) rearrangement is $90^{\circ}$ rotation of a single carboncarbon bond which can lead to the formation of two 5-membered rings and two 7 -membered rings 62. The SW rearrangement is a common defect found in carbon nanomaterials and it has been proposed that a localised vibrational mode associated with the defect may form the basis for monitoring the formation of these defects in nanotube growth [63. Consequently, it is of interest to study 

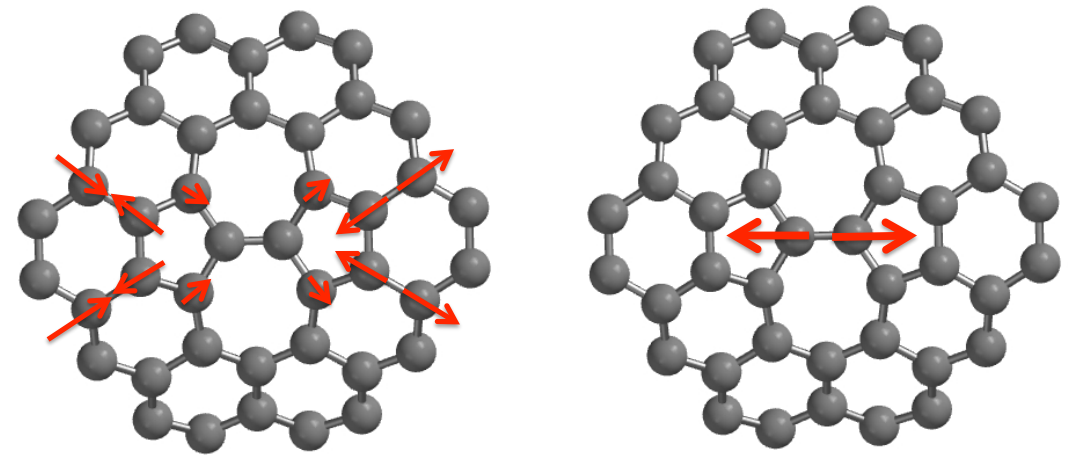

Figure 7: Illustration of the local vibrational modes associated with a Stone-Wales defect.

395

how the presence of these defects is reflected in the Raman spectroscopy.

For CNTs the inclusion of a single defect leads to some distinct highly localized modes based purely around the defect area. These defects are illustrated in Figure 7 and the computed Raman spectra for nanotubes and graphene are 00 shown in Figure 8. The first type of vibrational mode lies at $1665 \mathrm{~cm}^{-1}$ in a $(10,10)$ nanotube and is associated with stretching modes of the pentagon defect. The second corresponds to the C-C stretching mode between the two pentagon defects and is calculated to lie at $1931 \mathrm{~cm}^{-1}$ in a $(10,10)$ nanotube. The computed frequency of this mode is consistent with previous work 63 where the C-C stretching mode between the pentagon defects was computed to lie at $1962 \mathrm{~cm}^{-1}$ in DFT based calculations on a narrower diameter (3,3) nanotube. The computed spectra show the effect of the presence of the SW defect on the computed Raman spectra. The frequency of the RBM is not affected by the inclusion of the SW defect, and we find that the variation of the RBM frequency with diameter is also unaffected. The invariance of the RBM frequency to the presence of a SW defect was also observed in periodic tight binding density functional theory based calculations on a $(7,0)$ nanotube 29 . However, the Raman intensity of this mode is decreased significantly, and the single RBM becomes a collection of Raman active modes in the position of the original RBM, although 

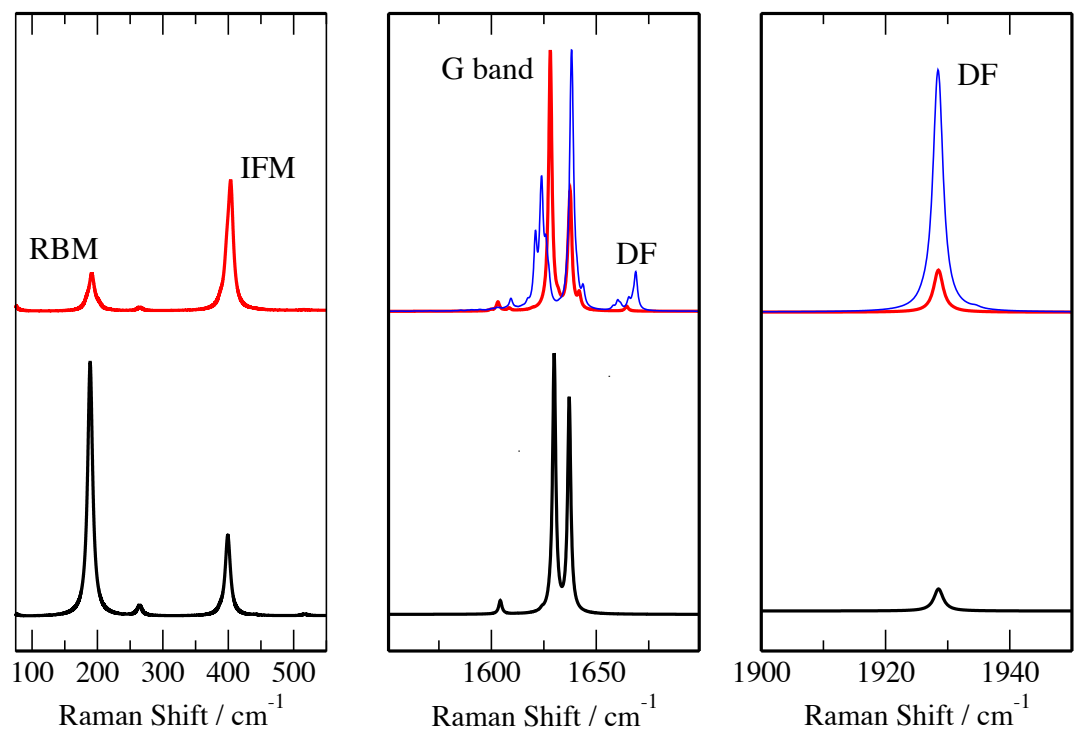

Figure 8: Effect of Stone-Wales defect on the computed Raman spectra for a $(10,10)$ nanotube. Black line: pristine nanotube, red line: one Stone-Wales defect, blue line: four Stone-Wales defects.

these modes are not resolved after the Lorentzian broadening, reflecting what is mostly likely observed experimentally. The frequency of the IFM is also unchanged, but the intensity of this mode increases with the presence of the defect.

In the $\mathrm{G}$ band region of the spectrum the SW defect leads to a new feature in the Raman spectrum. This defect band (labelled DF) lies at about $30 \mathrm{~cm}^{-1}$ higher in wavenumber than the $\mathrm{G}$ band and the intensity of this band increases as more SW defects are included in the nanotube. The spectra for this band have been generated with a narrower Lorentzian function $\left(2 \mathrm{~cm}^{-1}\right)$ so it can be seen more clearly. This feature arises from the vibrational modes associ425 ated with the pentagons and has been observed in previous studies [34, 29]. At higher frequency a band associated with the $\mathrm{C}-\mathrm{C}$ bond between the pentagons is evident. In the pristine nanotube there is a small band at this frequency associated with vibrational modes at the open ends of the nanotube. However, 


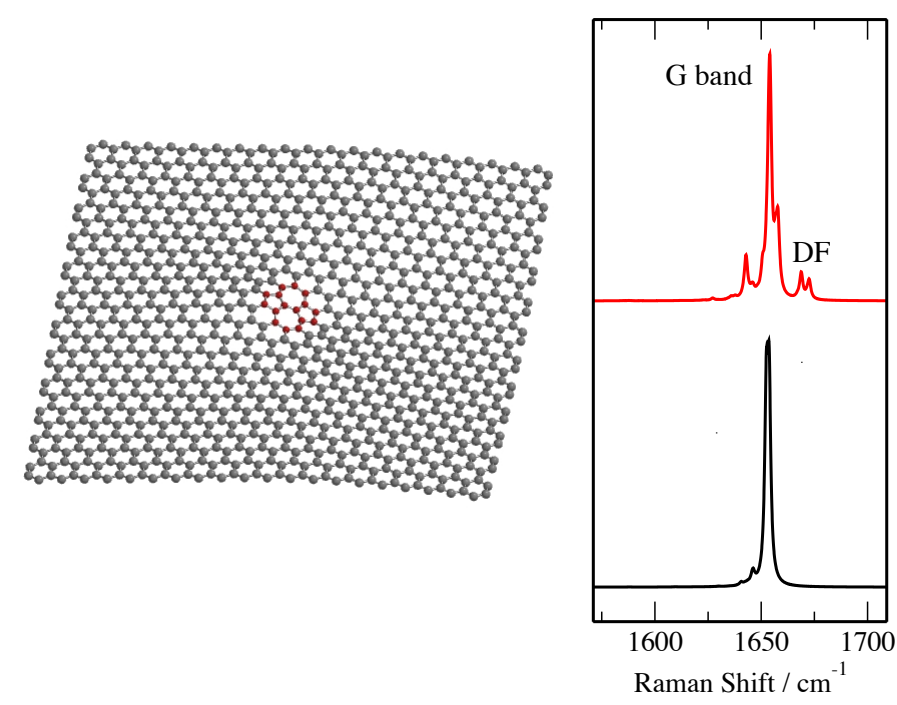

Figure 9: Effect of Stone-Wales defect on the Raman spectrum of graphene. Black line: pristine graphene, red line: one Stone-Wales defect.

the introduction of the SW defects leads to significant increase in the intensity

of the band at this frequency, and overall this band has a similar intensity to the IFM. For graphene, the inclusion of a SW defect can lead to buckling in a graphene sheet. The computed Raman spectra for a graphene flake are shown in Figure 9. The pentagon defects lead to an additional band at higher energy than the $\mathrm{G}$ band, although the separation is $15 \mathrm{~cm}^{-1}$ which is smaller than for the nanotube and so this band is less distinct from the $\mathrm{G}$ band. The $\mathrm{C}-\mathrm{C}$ stretching mode between the pentagons lies at $1922 \mathrm{~cm}^{-1}$ which is lower than calculated for the nanotube. The presence of these new bands in the Raman spectroscopy of nanotubes can provide evidence for the presence of SW defects in the sample.

440

\subsection{Nanotube Junctions}

Junctions are points where two CNTs of two different chiralities meet. The junction modelled here, shown in Figure 10 , is non-concentric between $(10,10)$ 

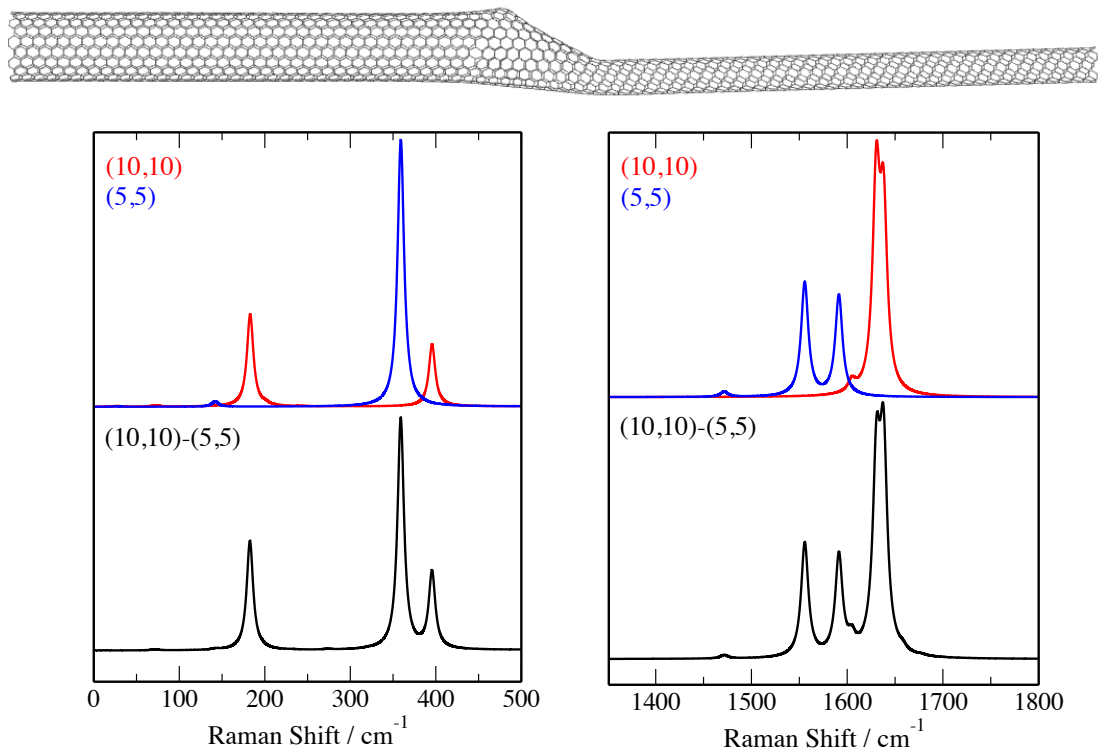

Figure 10: Computed Raman spectra for a nanotube junction, lower spectra: nanotube junction and upper spectra: individual nanotubes.

and $(5,5)$ nanotubes, and was generated via the ConTub v1.0 program [64. Figure 10 also shows the computed Raman spectra for the junction system in addition to the Raman spectra for the individual nanotubes. For this junction the separate nanotubes have distinct RBM and G bands, and the resulting Raman spectrum is very similar to the superposition of the Raman spectra for the individual tubes. This indicates that there is little coupling between the vibrational modes of the two nanotubes and they simply act as two independent nanotubes. Vibrational modes associated with the join region between the two CNTs lie between $1640-1670 \mathrm{~cm}^{-1}$ which lie in the tail of the $\mathrm{G}$ band, however it is difficult to differentiate them from the tail of the $\mathrm{G}$ band. The join region between the two nanotubes is constructed from hexagons and no pentagon defects are introduced. Consequently, bands associated with pentagon defects are not observed. 


\section{Conclusions}

The MM potential has been parameterised to model the vibrational spectroscopy of carbon-based materials such as CNTs and graphene via a MonteCarlo based minimisation of the RMSD of the DFT hessian matrix of $\mathrm{C}_{60}$ fullerene. When combined with the BPM the resulting potential provides a method to simulate the non-resonant Raman spectroscopy that can be applied to systems with thousands of atoms. For CNTs the computed Raman spectra accurately reproduce experimental observations. The known dependence of the RBM with the diameter of the nanotube and the nature of the vibrational modes corresponding to the $\mathrm{G}$ band are reproduced and a satisfactory approximation to the VDOS is obtained. The effect of SW defects on the Raman spectroscopy of CNTs and graphene has been explored, and new bands are observed that arise from new localised vibrational modes associated with the defect. CNT junctions have also been studied and it is shown that the resulting Raman spectra correspond to a superposition of the spectra for the individual nanotubes. Overall we have demonstrated that is it possible to simulate the vibrational spectroscopy of carbon nanomaterials using a fully empirical method, allowing accurate spectra to be calculated quickly for large systems which are not affordable with current 475 quantum chemical based methods.

\section{Acknowledgements}

The authors would like to thank the University of Nottingham for access to its High Performance Computing facility and the award of a studentship to PMT.

\section{References}

[1] S. Iijima, Helical microtubules of graphitic carbon, Nature 354 (6348) (1991) 56-58. doi:10.1038/354056a0. 
[2] K. S. Novoselov, A. K. Geim, S. V. Morozov, D. Jiang, Y. Zhang, S. V. Dubonos, I. V. Grigorieva, A. A. Firsov, Electric field effect in atomically thin carbon films, Science 306 (5696) (2004) 666-669. doi:10.1126/ science.1102896.

[3] M. S. Dresselhaus, G. Dresselhaus, R. Saito, A. Jorio, Raman spectroscopy of carbon nanotubes, Phys. Rep. 409 (2) (2005) 47. doi:10.1016/j. physrep.2004.10.006

[4] L. M. Malard, M. A. Pimenta, G. Dresselhaus, M. S. Dresselhaus, Raman spectroscopy in graphene, Physics Reports 473 (5-6) (2009) 51-87. doi: $10.1016 / \mathrm{j}$.physrep.2009.02.003

[5] A. Jorio, M. A. Pimenta, A. G. S. Filho, R. Saito, G. Dresselhaus, M. S. Dresselhaus, Characterizing carbon nanotube samples with resonance raman scattering, New Journal of Physics 5 (1) (2003) 139. doi: $10.1088 / 1367-2630 / 5 / 1 / 139$

[6] C. H. Choi, M. Kertesz, L. Mihaly, Vibrational assignment of all 46 fundamentals of $\mathrm{C}_{60}$ and $\mathrm{C}_{60}^{6-}$ : A scaled quantum mechanical results performed in redundant internal coordinates and compared to experiments, The Journal of Physical Chemistry A 104 (1) (2000) 102-112. doi:10.1021/jp991420h

[7] V. Schettino, M. Pagliai, L. Ciabini, G. Cardini, The vibrational spectrum of fullerene $\mathrm{C}_{60}$, The Journal of Physical Chemistry A 105 (50) (2001) 11192-11196. doi:10.1021/jp012874t.

[8] V. Schettino, M. Pagliai, G. Cardini, The infrared and Raman spectra of fullerene $\mathrm{C}_{70}$. DFT calculations and correlation with $\mathrm{C}_{60}$, The Journal of Physical Chemistry A 106 (9) (2002) 1815-1823. doi:10.1021/jp012680d.

[9] G. Sun, M. Kertesz, Vibrational Raman spectra of $\mathrm{C}_{70}$ and $\mathrm{C}_{60}{ }^{6-}$ studied by density functional theory, The Journal of Physical Chemistry A 106 (26) (2002) 6381-6386. doi:10.1021/jp020222e. 
[12] H. Chen, M. R. Golder, F. Wang, S. K. Doorn, R. Jasti, S. Tretiak, A. K. Swan, Raman-active modes of even-numbered cycloparaphenylenes: Com-

[14] M. Aydin, D. L. Akins, Calculated dependence of vibrational band frequencies of single-walled and double-walled carbon nanotubes on diameter, Vi-

[15] T. Kupka, M. Stachw, E. Chemecka, K. Pasterny, L. Stobiski, DFT calculations of structures, ${ }^{13} \mathrm{C}$ NMR chemical shifts and Raman RBM mode of simple models of ultra small diameter $(4,0)$ zigzag hydroxylated sin535 gle wall carbon nanotubes, Synthetic Metals 162 (78) (2012) 573 - 583. doi:10.1016/j.synthmet.2012.02.002.

[16] T. Yumura, D. Nozaki, S. Bandow, K. Yoshizawa, S. Iijima, End-cap effects on vibrational structures of finite-length carbon nanotubes, Journal of the 

ja0522579.

[17] T. Kupka, M. Stachow, L. Stobinski, J. Kaminsky, Calculation of raman parameters of real-size zigzag $(\mathrm{n}, 0)$ single-walled carbon nanotubes using finite-size models, Phys. Chem. Chem. Phys. 18 (2016) 25058-25069. doi: 10.1039/C6CP04100K

[18] W. A. Saidi, Effects of topological defects and diatom vacancies on characteristic vibration modes and raman intensities of zigzag single-walled carbon nanotubes, The Journal of Physical Chemistry A 118 (35) (2014) 7235-7241. doi:10.1021/jp409209s

[19] S. Guha, J. Menéndez, J. Page, G. Adams, Empirical bond polarizability 550 model for fullerenes, Physical Review B 53 (19) (1996) 13106-13114. doi: 10.1103/PhysRevB.53.13106

[20] J. Kapitán, M. Dračínský, J. Kaminský, L. Benda, P. Bouř, Theoretical modeling of magnesium ion imprints in the raman scattering of water,

घ The Journal of Physical Chemistry B 114 (10) (2010) 3574-3582. doi: 10.1021/jp9110508

[21] B. Nikoli, I. Miloevi, M. Damnjanovi, Raman intensities of totally symmetrical modes of homogeneously deformed single-walled carbon nanotubes, The Journal of Physical Chemistry C 118 (35) (2014) 20576-20584. doi:10.1021/jp503109b.

[22] D. Kahn, J. P. Lu, Vibrational modes of carbon nanotubes and nanoropes, Phys. Rev. B 60 (1999) 6535-6540. doi:10.1103/PhysRevB.60.6535.

[23] H. A. Witek, S. Irle, G. Zheng, W. A. de Jong, K. Morokuma, Modeling carbon nanostructures with the self-consistent charge density-functional tight-binding method: Vibrational spectra and electronic structure of $\mathrm{C}_{28}$, 565 ㄴ $\mathrm{C}_{60}$, and $\mathrm{C}_{70}$, The Journal of Chemical Physics 125 (21). doi:10.1063/1. 2370877. 
[24] R. Saito, T. Takeya, T. Kimura, G. Dresselhaus, M. S. Dresselhaus, Raman intensity of single-wall carbon nanotubes, Phys. Rev. B 57 (1998) 41454153. doi:10.1103/PhysRevB.57.4145.

[25] A. Rahmani, J.-L. Sauvajol, S. Rols, C. Benoit, Nonresonant Raman spectrum in infinite and finite single-wall carbon nanotubes, Phys. Rev. B 66 (2002) 125404. doi:10.1103/PhysRevB.66.125404

[26] G. Wu, J. Zhou, J. Dong, Raman modes of the deformed single-wall carbon nanotubes, Phys. Rev. B 72 (2005) 115411. doi:10.1103/PhysRevB.72. 115411

[27] K. Sbai, A. Rahmani, , H. Chadli, J.-L. Bantignies, P. Hermet, J.-L. Sauvajol, Infrared spectroscopy of single-walled carbon nanotubes, The

口 Journal of Physical Chemistry B 110 (25) (2006) 12388-12393. doi: 10.1021/jp0574504

[28] V. N. Popov, L. Henrard, P. Lambin, Electron-phonon and electron-photon interactions and resonant Raman scattering from the radial-breathing mode

口 of single-walled carbon nanotubes, Phys. Rev. B 72 (2005) 035436. doi: 10.1103/PhysRevB.72.035436.

[29] V. N. Popov, P. Lambin, Theoretical resonant Raman spectra of nanotube $(7,0)$ with point defects, physica status solidi (b) 246 (11-12) (2009) 26022605. doi:10.1002/pssb.200982279.

[30] D. W. Brenner, O. A. Shenderova, J. A. Harrison, S. J. Stuart, B. Ni, S. B. Sinnott, A second-generation reactive empirical bond order (REBO) potential energy expression for hydrocarbons, Journal of Physics: Condensed Matter 14 (4) (2002) 783-802. doi:10.1088/0953-8984/14/4/312

[31] D. W. Brenner, Empirical potential for hydrocarbons for use in simulating the chemical vapor deposition of diamond films, Phys. Rev. B 42 (1990) 9458-9471. doi:10.1103/PhysRevB.42.9458. 
[32] J. Tersoff, Empirical interatomic potential for carbon, with applications to amorphous carbon, Phys. Rev. Lett. 61 (1988) 2879-2882. doi:10.1103/ PhysRevLett.61.2879

[33] A. C. T. Van Duin, S. Dasgupta, F. Lorant, W. A. Goddard, ReaxFF: A reactive force field for hydrocarbons, Journal of Physical Chemistry A 105 (41) (2001) 9396-9409. doi:10.1021/jp004368u

[34] G. Wu, J. Dong, Raman characteristic peaks induced by the topological defects of carbon nanotube intramolecular junctions, Phys. Rev. B 73 (2006) 245414. doi:10.1103/PhysRevB.73.245414.

[35] T. Takai, C. Lee, T. Halicioglu, W. A. Tiller, A model potential function for carbon systems: Clusters, J. Phys. Chem. 94 (8) (1990) 4480-4482. doi:10.1021/j100374a025.

[36] J. N. Murrell, R. E. Mottram, Potential energy functions for atomic 口 solids, Molecular Physics 69 (3) (1990) 571-585. doi:10.1080/ 00268979000100411

[37] S. P. Balm, A. W. Allaf, H. W. Kroto, J. N. Murrell, Potential-energy function for large carbon clusters, J. Chem. Soc., Faraday Trans. 87 (1991) 803-806. doi:10.1039/FT9918700803.

[38] B. R. Eggen, R. L. Johnston, J. N. Murrell, Carbon cluster structures and stabilities predicted from solid-state potentials, Journal of the Chem-

1 ical Society, Faraday Transactions 90 (20) (1994) 3029. doi:10.1039/ ft9949003029.

[39] H. Do, N. A. Besley, Calculation of the vibrational frequencies of carbon clusters and fullerenes with empirical potentials, Physical Chemistry Chemical Physics 17 (5) (2015) 3898-3908. doi:10.1039/C4CP05424E

[40] R. L. Johnston, J.-Y. Fang, An empirical many body potential energy function for aluminum. application to solid phases and microclusters, The 
Journal of Chemical Physics 97 (10) (1992) 7809-7821. doi:10.1063/1. 463450 .

[41] J.-Y. Fang, R. L. Johnston, J. N. Murrell, Potential-energy functions for $\mathrm{Cu}, \mathrm{Ag}$ and $\mathrm{Au}$ solids and their application to clusters of these ele-

625 ments, J. Chem. Soc., Faraday Trans. 89 (1993) 1659-1665. doi:10.1039/ FT9938901659.

[42] F. Gao, R. L. Johnston, J. N. Murrell, Empirical many-body potential energy functions for iron, The Journal of Physical Chemistry 97 (46) (1993) 12073-12082. doi:10.1021/j100148a038.

[43] N. A. Besley, R. L. Johnston, A. J. Stace, J. Uppenbrink, Theoretical study of the structures and stabilities of iron clusters, Journal of Molecular Structure: THEOCHEM 341 (1) (1995) 75 - 90. doi:10.1016/0166-1280(95) 04207-M.

[44] J. E. Hearn, R. L. Johnston, S. Leoni, J. N. Murrell, Global potentials for calcium and strontium solids, J. Chem. Soc., Faraday Trans. 92 (1996) 425-432. doi:10.1039/FT9969200425.

[45] L. D. Lloyd, R. L. Johnston, Modelling aluminium clusters with an empirical many-body potential, Chemical Physics 236 (13) (1998) 107 - 121. doi:10.1016/S0301-0104(98)00180-3.

[46] H. Cox, R. L. Johnston, A. Ward, An empirical many-body potential energy function for modelling ytterbium, Journal of Physics: Condensed Matter $10(42)(1998) 9419$.

[47] H. Cox, R. L. Johnston, J. N. Murrell, Empirical potentials for modeling solids, surfaces, and clusters, Journal of Solid State Chemistry 145 (2) (1999) 517-540. doi:doi:10.1006/jssc.1999.8200.

[48] F. Ercolessi, J. B. Adams, Interatomic potentials from first-principles calculations: The force-matching method, Europhysics Letters 26 (8) (1994) 583-588. doi:10.1209/0295-5075/26/8/005. 
[49] H. Do, A. Troisi, Developing accurate molecular mechanics force fields for conjugated molecular systems, Phys. Chem. Chem. Phys. 17 (2015) 2512325132. doi:10.1039/C5CP04328J.

[50] S. Grimme, A general quantum mechanically derived force field (QMDFF) for molecules and condensed phase simulations, Journal of Chemical Theory and Computation 10 (10) (2014) 4497-4514. doi:10.1021/ct500573f.

[51] A. D. Becke, Density-functional thermochemistry. iii. The role of exact exchange, The Journal of Chemical Physics 98 (7) (1993) 5648-5652. doi: 10.1063/1.464913.

[52] P. J. Stephens, F. J. Devlin, C. F. Chabalowski, M. J. Frisch, Ab initio calculation of vibrational absorption and circular dichroism spectra using density functional force fields, The Journal of Physical Chemistry 98 (45) (1994) 11623-11627. doi:10.1021/j100096a001.

[53] J. P. Merrick, D. Moran, L. Radom, An evaluation of harmonic vibrational frequency scale factors, Journal of Physical Chemistry A 111 (45) (2007) 11683-11700. doi:10.1021/jp073974n.

[54] R. Saito, M. Furukawa, G. Dresselhaus, M. S. Dresselhaus, Raman spectra of graphene ribbons., Journal of physics. Condensed matter 22 (33) (2010) 334203. doi:10.1088/0953-8984/22/33/334203.

[55] R. Saito, T. Takeya, T. Kimura, G. Dresselhaus, M. S. Dresselhaus, Raman intensity of single-wall carbon nanotubes, Phys. Rev. B 57 (7) (1998) 41454153. doi:10.1103/PhysRevB.57.4145.

[56] Y. Shao, Z. Gan, E. Epifanovsky, A. T. B. Gilbert, M. Wormit, J. Kussmann, A. W. Lange, A. Behn, J. Deng, X. Feng, D. Ghosh, M. Goldey, P. R. Horn, L. D. Jacobson, I. Kaliman, R. Z. Khaliullin, T. Ku, A. Landau, J. Liu, E. I. Proynov, Y. M. Rhee, R. M. Richard, M. A. Rohrdanz, R. P. Steele, E. J. Sundstrom, H. L. W. III, P. M. Zimmerman, D. Zuev, B. Albrecht, E. Alguire, B. Austin, G. J. O. Beran, Y. A. Bernard, E. Berquist, 
K. Brandhorst, K. B. Bravaya, S. T. Brown, D. Casanova, C.-M. Chang, Y. Chen, S. H. Chien, K. D. Closser, D. L. Crittenden, M. Diedenhofen, R. A. D. Jr., H. Do, A. D. Dutoi, R. G. Edgar, S. Fatehi, L. Fusti-Molnar, A. Ghysels, A. Golubeva-Zadorozhnaya, J. Gomes, M. W. Hanson-Heine, P. H. Harbach, A. W. Hauser, E. G. Hohenstein, Z. C. Holden, T.-C. Jagau, H. Ji, B. Kaduk, K. Khistyaev, J. Kim, J. Kim, R. A. King, P. Klunzinger, D. Kosenkov, T. Kowalczyk, C. M. Krauter, K. U. Lao, A. D. Laurent, K. V. Lawler, S. V. Levchenko, C. Y. Lin, F. Liu, E. Livshits, R. C. Lochan, A. Luenser, P. Manohar, S. F. Manzer, S.-P. Mao, N. Mardirossian, A. V. Marenich, S. A. Maurer, N. J. Mayhall, E. Neuscamman, C. M. Oana, R. Olivares-Amaya, D. P. ONeill, J. A. Parkhill, T. M. Perrine, R. Peverati, A. Prociuk, D. R. Rehn, E. Rosta, N. J. Russ, S. M. Sharada, S. Sharma, D. W. Small, A. Sodt, T. Stein, D. Stck, Y.-C. Su, A. J. Thom, T. Tsuchimochi, V. Vanovschi, L. Vogt, O. Vydrov, T. Wang, M. A. Watson, J. Wenzel, A. White, C. F. Williams, J. Yang, S. Yeganeh, S. R. Yost, Z.-Q. You, I. Y. Zhang, X. Zhang, Y. Zhao, B. R. Brooks, G. K. Chan, D. M. Chipman, C. J. Cramer, W. A. G. III, M. S. Gordon, W. J. Hehre, A. Klamt, H. F. S. III, M. W. Schmidt, C. D. Sherrill, D. G. Truhlar, A. Warshel, X. Xu, A. Aspuru-Guzik, R. Baer, A. T. Bell, N. A. Besley, J.-D. Chai, A. Dreuw, B. D. Dunietz, T. R. Furlani, S. R. Gwaltney, C.-P. Hsu, Y. Jung, J. Kong, D. S. Lambrecht, W. Liang, C. Ochsenfeld, V. A. Rassolov, L. V. Slipchenko, J. E. Subotnik, T. V. Voorhis, J. M. Herbert, A. I. Krylov, P. M. Gill, M. Head-Gordon, Advances in molecular quantum chemistry contained in the Q-Chem 4 program package, Molecular Physics 113 (2) (2015) 184-215. doi:10.1080/00268976.2014.952696

[57] M. S. Dresselhaus, A. Jorio, A. G. Souza Filho, R. Saito, Defect characterization in graphene and carbon nanotubes using Raman spectroscopy, Philosophical Transactions of the Royal Society A: Mathematical, Physical and Engineering Sciences 368 (1932) (2010) 5355-5377. doi:10.1098/ rsta.2010.0213. 
[58] C. Fantini, A. Jorio, M. Souza, M. S. Strano, M. S. Dresselhaus, M. A. Pimenta, Optical transition energies for carbon nanotubes from resonant Raman spectroscopy: Environment and temperature effects, Phys. Rev. Lett. 93 (2004) 147406. doi:10.1103/PhysRevLett.93.147406.

[59] A. G. Souza Filho, S. G. Chou, G. G. Samsonidze, G. Dresselhaus, M. S. Dresselhaus, L. An, J. Liu, A. K. Swan, M. S. Ünlü, B. B. Goldberg, A. Jorio, A. Grüneis, R. Saito, Stokes and anti-stokes Raman spectra of small-diameter isolated carbon nanotubes, Phys. Rev. B 69 (2004) 115428. doi:10.1103/PhysRevB.69.115428

[60] J. Maultzsch, H. Telg, S. Reich, C. Thomsen, Radial breathing mode of single-walled carbon nanotubes: Optical transition energies and chiralindex assignment, Physical Review B - Condensed Matter and Materials Physics 72 (20) (2005) 1-16. doi:10.1103/PhysRevB.72.205438.

[61] M. S. Dresselhaus, P. C. Eklund, Phonons in carbon nanotubes, Adv. Phys. 49 (2000) 705-814. doi:10.1080/000187300413184.

[62] A. Stone, D. Wales, Theoretical studies of icosahedral $\mathrm{C}_{60}$ and some related species, Chemical Physics Letters 128 (5) (1986) $501-503$.

[63] Y. Miyamoto, A. Rubio, S. Berber, M. Yoon, D. Tománek, Spectroscopic characterization of stone-wales defects in nanotubes, Phys. Rev. B 69 (2004) 121413. doi:10.1103/PhysRevB.69.121413.

[64] S. Melchor, J. A. Dobado, ConTub: An algorithm for connecting two arbitrary carbon nanotubes, Journal of Chemical Information and Computer Sciences 44 (5) (2004) 1639-1646. doi:10.1021/ci049857w. 